\title{
ON THE PHI AND PSI TRANSFORMS OF FRAZIER AND JAWERTH
}

\author{
H.-Q. Bui ${ }^{1}$ and M. Paluszyński ${ }^{2}$ \\ ${ }^{1}$ Department of Mathematics \& Statistics, \\ University of Canterbury, \\ Private Bag 4800, Christchurch, New Zealand. \\ ${ }^{2}$ Institute of Mathematics, \\ University of Wroclaw, \\ 50-384 Wroclaw, Poland.
}

Report Number: UCDMS2004/11 May 2004

Keywords: Triebel-Lizorkin spaces, Calderón reproducing formula, spanning, completeness, density. 



\title{
ON THE PHI AND PSI TRANSFORMS OF FRAZIER AND JAWERTH
}

\author{
HUY-QUI BUI AND MACIEJ PALUSZYŃSKI
}

\begin{abstract}
In this article we study the phi and psi tranforms of Frazier and Jawerth when either phi or psi is not band-limited. Our main result concerns the invertibility of these transforms and is used to show that certain affine system generated by the Mexican hat function is complete in Lebesgue and Hardy spaces.

2000 AMS Classification: 42B25, 42C30, 46E35.
\end{abstract}

The phi and psi transforms were developed by M. Frazier and B. Jawerth $[8,7]$, in parallel with the well-known wavelet theory. Their principal aim was to obtain and then to study the decomposition of a function or a distribution $f$ in the form $f=\sum_{Q}\left\langle f, \varphi_{Q}\right\rangle \psi_{Q}$, where the sum is taken over the family of all dyadic cubes in $\mathbf{R}^{n}$. In the notation of wavelet theory, this decomposition takes the form

$$
f=\sum_{j \in \mathbf{Z}, k \in \mathbf{Z}^{n}}\left\langle f, \varphi_{j, k}\right\rangle \psi_{j, k} .
$$

(See $\S 1$ for the relevant notations).

A most satisfactory theory was obtained by the authors when both $\varphi$ and $\psi$ are in $\mathcal{S}$, and satisfy certain additional conditions, including the condition that $\varphi$ and $\psi$ have their Fourier transforms supported in an annulus about the origin (and hence both are band-limited and have infinitely many vanishing moments). The important feature of this theory is that the norm of $f$ (in various function spaces) is equivalent to an appropriate norm of the sequence $\left\{\left\langle f, \varphi_{j, k}\right\rangle\right\}=S_{\varphi}(f)$, the phi transform of $f$. The decomposition $(*)$ then allows one to recover $f$ from its "coefficients" $\left\{\left\langle f, \varphi_{j, k}\right\rangle\right\}$ and the "building blocks" $\left\{\psi_{j, k}\right\}$.

In this article we study the non-bandlimited case of the phi and psi transforms and aim also to simplify the original proofs given in the fundamental work [8]. We treat these transforms separately and aim to find simply stated conditions to ensure their boundedness on various function spaces. By doing so we could see more transparently the conditions which imply the boundedness of these transforms. As it turns out this requires only an appropriate moment condition on the

The second author was supported by a Polish research grant KBN 5P03A05020. 
generating function phi or psi: The moment condition obtained for phi is minimal, and that for psi is the same as in [8]. When $\psi$ is bandlimited, the exact reconstruction $(*)$ is a consequence of a "Tauberian condition" (see Theorem 3.1 and [8, Theorem 2.2]). However, when $\psi$ is not band-limited, the result corresponding to $(*)$ is less satisfactory (see Theorem 3.2 and [8, Theorem 4.2]); one can only establish the invertibility of an operator corresponding to the right-hand side of $(*)$. This is due to the lack of a good replacement for a sampling theorem by Frazier and Jawerth [7, Lemma 2.1].

Although we follow the general strategy of the proofs in [8], we use some recent results in $[2,4]$ which allow us to deal directly with the case where phi or psi is not band-limited. Moreover, the separation of the moment condition and the Tauberian condition has its root in the works $[2,3]$ by the authors and M.H. Taibleson. The results from the Frazier-Jawerth approach that we will use are of a real-variable nature: a Peetre-Stein typeestimate for sequence spaces (see Lemma 1.1), and a Calderón type sampling formula for distributions ([7, Lemma 2.1]).

Another novelty of our work lies in the proof of Theorem 3.2, in which we avoid the use of the molecular decompositions of the TriebelLizorkin spaces in [8] and, consequently, we obtain a relatively simple proof of the corresponding result in that paper. Theorem 3.2 is the main result in this paper and is motivated by our attempt to solve an $L^{p}$-spanning problem for the Mexican hat function posed by Y. Meyer in [11] (see Theorem 3.3). Further discussion of our results with those in [8] and other results in the literature will be given in the relevant sections.

This paper may be considered as a part in the group of papers [2][4], of which it is the final one. The aim was to study function spaces in which the "kernel" function is not band-limited. As such, this series of papers bridges the gap between the classical theory, in which the Poisson kernel or the Gaussian kernel is used, and the more recent Littlewood-Paley method where the kernel function is band-limited. We hope that this series may also serve as an independent introduction to some aspects of the theory of function spaces.

Acknowledgments. This work grew out of discussions the authors have had with Professor Guido Weiss at Washington University during 1999. They would like to thank him for helpful comments and suggestions. The authors also acknowledge the generous support, by Professor Weiss and colleagues in the Mathematics Department at Washington University, which has made their stay in St. Louis possible. 


\section{Preliminaries}

The aim of this section is to introduce the notation and the relevant definitions, as well as to state three basic results to be used in the proofs of our main theorems.

We use the standard notation regarding Lebesgue spaces, the Fourier transforms and distributions. All functions and distributions are defined on $\mathbf{R}^{n}$, so that explicit reference to $\mathbf{R}^{n}$ in the notation will usually be dropped. Thus, the Schwartz class is denoted by $\mathcal{S}$ and its dual, the space of tempered distributions, by $\mathcal{S}^{\prime}$. As usual $\|\cdot\|_{p}$ denotes the $L^{p}$-norm, and $\langle\cdot, \cdot\rangle$ denotes the inner product on $L^{2}$. The set of polynomials is denoted by $\mathcal{P}$.

First we give the definitions of certain sequence spaces following M. Frazier and B. Jawerth [8]. However, instead of the family of dyadic cubes, we shall use $\left\{(j, k): j \in \mathbf{Z}, k \in \mathbf{Z}^{n}\right\}$ as the index set for sequences.

For $-\infty<\alpha<\infty, 0<p<\infty, 0<q \leq \infty$, and a sequence $s=\left\{s_{j, k}\right\}_{j \in \mathbf{Z}, k \in \mathbf{Z}^{n}}$, we define

$$
\|s\|_{\dot{\mathbf{f}}_{p, q}^{\alpha}}=\left\|\left(\sum_{j \in \mathbf{Z}} \sum_{k \in \mathbf{Z}^{n}}\left(2^{j \alpha}\left|s_{j, k}\right| \chi_{j, k}\right)^{q}\right)^{1 / q}\right\|_{p},
$$

where $\chi=\chi_{Q_{0,0}}$ is the characteristic function of the unit cube $Q_{0,0}=$ $[0,1) \times \cdots \times[0,1)$, and adopting the notation in wavelet theory we let

$$
\chi_{j, k}(x)=2^{j n / 2} \chi\left(2^{j} x-k\right) .
$$

Note that $\chi_{j, k}=2^{j n / 2} \chi_{Q_{j, k}}$, where $\chi_{Q_{j, k}}$ is the characteristic function of the cube

$Q_{j, k}=2^{-j}\left(Q_{0,0}+k\right)=\left[k_{1} 2^{-j},\left(k_{1}+1\right) 2^{-j}\right) \times \cdots \times\left[k_{n} 2^{-j},\left(k_{n}+1\right) 2^{-j}\right)$.

We define $\dot{\mathbf{f}}_{p, q}^{\alpha}$ to be the space of all sequences $s$ for which $\|s\|_{\dot{\mathbf{f}}_{p, q}^{\alpha}}$ is finite.

The letters $\varphi$ and $\psi$ will be used to denote functions in $\mathcal{S}$. Additional assumptions on $\varphi$ and $\psi$ will be stated when required. For $f \in \mathcal{S}^{\prime}$, we define a sequence $S_{\varphi}(f)$ by

$$
S_{\varphi}(f)=\left\{S_{j, k}(f, \varphi)\right\}=\left\{\left\langle f, \varphi_{j, k}\right\rangle\right\},
$$

where $\varphi_{j, k}$ is similarly defined to $(1.2),\left\langle f, \varphi_{j, k}\right\rangle=\left(f, \overline{\varphi_{j, k}}\right)$, and $(\cdot, \cdot)$ denotes the pairing between a distribution and a test function. For $j \in \mathbf{Z}$ and $k \in \mathbf{Z}^{n}$, we let $\varphi_{j}(x)=2^{j n} \varphi\left(2^{j} x\right)$, and

$$
M_{\varphi}(f)=\left\{M_{j, k}(f, \varphi)\right\}=\left\{2^{-j n / 2} \sup _{y \in Q_{j, k}}\left|\left(\varphi_{j} * f\right)(y)\right|\right\},
$$


Let $\lambda>0$. For every $x \in Q_{j, k}$, it is easy to see that

$$
\begin{aligned}
M_{j, k}(f, \varphi) & \approx 2^{-j n / 2} \sup _{y \in Q_{j, k}}\left|\left(\varphi_{j} * f\right)(y)\right|\left(1+2^{j}|x-y|\right)^{-\lambda} \\
& \leq 2^{-j n / 2} \varphi_{j, \lambda}^{*} f(x)
\end{aligned}
$$

where

$$
\varphi_{j, \lambda}^{*} f(x)=\sup _{y \in \mathbf{R}^{n}}\left|\left(\varphi_{j} * f\right)(y)\right|\left(1+2^{j}|x-y|\right)^{-\lambda}
$$

is the Peetre's maximal function.

Let $\lambda>0$ and $0<r<\infty$. For a sequence $s$, we define its "maximal sequence" $s^{*}=s^{*}(r, \lambda)=\left\{s_{j, k}^{*}\right\}$ by

$$
s_{j, k}^{*}=\left(\sum_{m \in \mathbf{Z}^{n}}\left|s_{j, m}\right|^{r}(1+|k-m|)^{-\lambda}\right)^{1 / r} .
$$

Clearly, $\left|s_{j, k}\right| \leq s_{j, k}^{*}$ for all $j, k$.

REMARK. As mentioned above we have changed the notation in [8] where the sequences are indexed by the dyadic cubes. The sequence $M_{\varphi}(f)$ is denoted by $\sup (f)$ in [8].

We next recall the definition of the homogeneous Triebel-Lizorkin spaces ([12]). Let $-\infty<\alpha<\infty, 0<p<\infty$ and $0<q \leq \infty$. Let $\varphi \in \mathcal{S}$ be $\operatorname{such}$ that $\operatorname{supp}(\hat{\varphi}) \subseteq\{1 / 2 \leq|\xi| \leq 2\}$, and

$$
\sum_{j \in \mathbf{Z}}\left(\hat{\varphi}\left(2^{j} \xi\right)\right)^{2}=1 \quad \text { for all } \xi \neq 0 .
$$

We define the homogeneous Triebel-Lizorkin spaces by

$$
\dot{F}_{p, q}^{\alpha}=\left\{f \in \mathcal{S}^{\prime} / \mathcal{P}:\|f\|_{\dot{F}_{p, q}^{\alpha}}=\left\|\left(\sum_{j \in \mathbf{Z}}\left(2^{j \alpha}\left|\varphi_{j} * f\right|\right)^{q}\right)^{1 / q}\right\|_{p}<\infty\right\},
$$

with the usual interpretation when $q=\infty$.

It is well-known that the spaces $\dot{F}_{p, q}^{\alpha}$ are independent of the function $\varphi$ (satisfying the above conditions), and that they contain Lebesgue spaces, Hardy spaces and Sobolev spaces as special cases (see e.g. [12, 9, 14]). Moreover, the characterization of these spaces, under "minimal" moment and Tauberian conditions on $\varphi$, has been proved in $[2,3]$.

We now state three results which form the necessary tools for the proofs of our theorems. The first is a norm-equivalence on sequence spaces by Frazier and Jawerth [8, Lemma 2.3] . 
Lemma 1.1. If $0<p<\infty, 0<q \leq \infty, \lambda>n$, and $r=\min (p, q)$, then we have the following norm-equivalence:

$$
\|s\|_{\dot{\mathbf{f}}_{p, q}^{\alpha}} \approx\left\|s^{*}\right\|_{\dot{\mathbf{f}}_{p, q}^{\alpha}}
$$

for every $-\infty<\alpha<\infty$

The second is an estimate of Heideman type, which is a slight variant of $[2$, Lemma 2.1 (Remark)]. Let $m \in \mathbf{Z}$, we say that $\eta \in \mathcal{S}$ has $m$ vanishing moments if

$$
\int_{\mathbf{R}^{n}} x^{\kappa} \eta(x) d x=0 \quad \text { for all }|\kappa| \leq m .
$$

When $m<0$, the above condition is vacuous.

Lemma 1.2. Let $A \geq 0, N_{1} \in \mathbf{R}, m \in \mathbf{Z}$ and $b>0$. Let $\varphi, \psi$ be in $\mathcal{S}$. Assume $\psi$ has $m$ vanishing moments and $N_{1}+A-1 \leq m$. Then there is a positive constant $C$ such that

$$
\left|\left(\psi_{t} * \varphi_{s}\right)(x)\right| \leq C s^{-n}\left(\frac{t}{s}\right)^{N_{1}}\left(1+\frac{|x|}{t}\right)^{-A}
$$

for all $x \in \mathbf{R}^{n}$, and $0<t \leq b s$, where $C=C\left(n, A, b, N_{1}\right) p_{N}(\psi) p_{N}(\varphi)$,

$$
p_{N}(\psi)=\sup _{|\kappa| \leq N, x \in \mathbf{R}^{n}}(1+|x|)^{N}\left|D^{\kappa} \psi(x)\right|
$$

for a sufficiently large non-negative integer $N$, and $p_{N}(\varphi)$ is similarly defined.

Proof. When $N_{1}+A-1=m$, the proof (for non-negative integers $\left.N_{1}, A\right)$ indicated in [2] works also for the case $A \geq 0, N_{1} \in \mathbf{R}$. If $N_{1}+A-1<m$, choose $A_{1}>A$ such that $N_{1}+A_{1}-1=m$. Then by the first case, we have

$$
\left|\left(\psi_{t} * \varphi_{s}\right)(x)\right| \leq C s^{-n}\left(\frac{t}{s}\right)^{N_{1}}\left(1+\frac{|x|}{t}\right)^{-A_{1}} .
$$

But $(1+|x| / t)^{-A_{1}} \leq(1+|x| / t)^{-A}$, so that the required inequality follows.

The third result is the discrete version of an estimate for the Peetre's maximal function in [2, Theorem 3.1].

Lemma 1.3. Let $-\infty<\alpha<\infty, 0<p<\infty, 0<q \leq \infty, \lambda>$ $\max (n / p, n / q)=n / \min (p, q)$. Assume that $\varphi \in \mathcal{S}$ has $[\alpha]$ vanishing moments. Then there is a positive constant $C$ such that

$$
\left\|\left(\sum_{j \in \mathbf{Z}}\left(2^{j \alpha} \varphi_{j, \lambda}^{*} f(x)\right)^{q}\right)^{1 / q}\right\|_{p} \leq C\|f\|_{\dot{F}_{p, q}^{\alpha}}
$$


for all $f \in \mathcal{S}^{\prime} / \mathcal{P}$.

REMARK. Since the proof of [2, Theorem 3.1] used an estimate similar to Lemma 1.2, the constant $C$ in Lemma 1.3 has the form $C=$ $C(n, \alpha, \lambda, p, q) p_{N}(\varphi)$ for a sufficiently large $N$.

\section{BOUNDEDNESS OF PHI AND PSI TRANSFORMS}

In this section we prove that the phi transform $S_{\varphi}$ is bounded under a "minimal" moment condition on $\varphi$. We then show that the psi transform $T_{\psi}$, which is a left inverse of $S_{\varphi}$ (under some aditional conditions on $\varphi$ and $\psi$ ), is also bounded. While the proof in [8, Theorem 2.2] is based on a certain norm-equivalence for entire functions of exponential type, our proof uses Lemma 1.1, Lemma 1.2 and Lemma 1.3, in which neither $\varphi$ nor $\psi$ is assumed to be band-limited.

Theorem 2.1. Let $-\infty<\alpha<\infty, 0<p<\infty$, and $0<q \leq \infty$. Assume that $\varphi \in \mathcal{S}$ has $[\alpha]$ vanishing moments. Then there is a positive constant $C$ such that

$$
\left\|M_{\varphi}(f)\right\|_{\dot{\mathbf{f}}_{p, q}^{\alpha}} \leq C\|f\|_{\dot{F}_{p, q}^{\alpha}}
$$

for all $f \in \mathcal{S}^{\prime} / \mathcal{P}$.

Proof. By (1.4) and (1.5),

$$
\left\|M_{\varphi}(f)\right\|_{\dot{\mathbf{f}}_{p, q}^{\alpha}} \leq C\left\|\left(\sum_{j \in \mathbf{Z}} \sum_{k \in \mathbf{Z}^{n}}\left(2^{j \alpha} 2^{-j n / 2} \chi_{j, k}(x) \varphi_{j, \lambda}^{*}(x)\right)^{q}\right)^{1 / q}\right\|_{p} .
$$

Since $\chi_{j, k}(x)=2^{j n / 2} \chi_{Q_{j, k}}(x)$, and for each $j \in \mathbf{Z}$ fixed, $\left\{Q_{j, k}\right\}_{k \in \mathbf{Z}^{n}}$ is a partition of $\mathbf{R}^{n}$, the sum with respect to $k$ in the right-hand side of the above is equal to $\left(2^{j \alpha} \varphi_{j, \lambda}^{*} f(x)\right)^{q}$. It follows that

$$
\begin{aligned}
\left\|M_{\varphi}(f)\right\|_{\dot{\mathbf{f}}_{p, q}^{\alpha}} & \leq C\left\|\left(\sum_{j \in \mathbf{Z}}\left(2^{j \alpha} \varphi_{j, \lambda}^{*} f(x)\right)^{q}\right)^{1 / q}\right\|_{p} \\
& \leq C\|f\|_{\dot{F}_{p, q}^{\alpha}}
\end{aligned}
$$

if $\lambda>\max (n / p, n / q)$ by Lemma 1.3.

Theorem 2.2. Let $\alpha, p, q$ and $\varphi$ be as in Theorem 2.1. Then the the map

$$
S_{\varphi}: \dot{F}_{p, q}^{\alpha} \rightarrow \dot{\mathbf{f}}_{p, q}^{\alpha}
$$

defined by (1.3) is bounded. 
Proof. Let $\tilde{\varphi}$ be the function defined by $\tilde{\varphi}(x)=\overline{\varphi(-x)}$. Then, for all $j, k$,

$$
\begin{aligned}
\left|\left\langle f, \varphi_{j, k}\right\rangle\right| & =\left|2^{-j n / 2}\left(\tilde{\varphi}_{j} * f\right)\left(k 2^{-j}\right)\right| \\
& \leq M_{j, k}(f, \tilde{\varphi}) .
\end{aligned}
$$

Since $\tilde{\varphi}$ also has $[\alpha]$ vanishing moments, the conclusion of the theorem follows from Theorem 2.1.

REMARK. By the remark to Lemma 1.3, the constant $C$ in Theorem 2.1 has the form $C=C(n, \alpha, p, q) p_{N}(\varphi)$ for a sufficiently large integer $N$, and hence a quantitative version of Theorem 2.2 is that the inequality

$$
\left\|S_{\varphi}(f)\right\|_{\dot{\mathbf{f}}_{p, q}^{\alpha}} \leq C(n, \alpha, p, q) p_{N}(\varphi)\|f\|_{\dot{F}_{p, q}^{\alpha}}
$$

holds for all $f \in \dot{F}_{p, q}^{\alpha}$.

We next consider another operator which corresponds to a left inverse of $S_{\varphi}$ when $\varphi$ satisfies some additional properties (besides the moment condition). Let $\psi$ be a function in $\mathcal{S}$. For a sequence $s \in \dot{\mathbf{f}}_{p, q}^{\alpha}$, we define

$$
T_{\psi}(s)=\sum_{j \in \mathbf{Z}} \sum_{k \in \mathbf{Z}^{n}} s_{j, k} \psi_{j, k}
$$

whenever the sum on the right-hand side of the above converges in $\mathcal{S}^{\prime} / \mathcal{P}$. We first show the convergence for a large class of functions $\psi \in \mathcal{S}$.

Lemma 2.3. Let $-\infty<\alpha<\infty, 0<p<\infty, 0<q \leq \infty$. If $\psi \in \mathcal{S}$ has $[n / \min (p, 1)-n-\alpha]$ vanishing moments, then the series defining $T_{\psi}(s)$ in (2.2) converges in $\mathcal{S}^{\prime} / \mathcal{P}$ for every $s \in \dot{\mathbf{f}}_{p, q}^{\alpha}$.

Proof. We shall prove the lemma by showing that

$$
\left(T_{\psi}(s), \varphi\right)=\sum_{j \in \mathbf{Z}} \sum_{k \in \mathbf{Z}^{n}} s_{j, k}\left(\psi_{j, k}, \varphi\right)
$$

converges absolutely for every $\varphi \in \mathcal{S}_{\infty}$, the space of functions in $\mathcal{S}$ having infinitely many vanishing moments, where $(\cdot, \cdot)$ denotes the pairing between a distribution and a test function. (Note that $\mathcal{S}_{\infty}^{\prime}=$ $\mathcal{S}^{\prime} / \mathcal{P}$.) 
First observe that, for each $j \in \mathbf{Z}$,

$$
\begin{aligned}
\|s\|_{\dot{\mathbf{f}}_{p, \infty}^{\alpha}} & =\left\|\sup _{l \in \mathbf{Z}, \mu \in \mathbf{Z}^{n}} 2^{l(\alpha+n / 2)}\left|s_{l, \mu}\right| \chi_{Q_{l, \mu}}(x)\right\|_{p} \\
& \geq\left\|\sup _{\mu \in \mathbf{Z}^{n}} 2^{j(\alpha+n / 2)}\left|s_{j, \mu}\right| \chi_{Q_{j, \mu}}(x)\right\|_{p} \\
& =\left\{\sum_{k \in \mathbf{Z}^{n}} \int_{Q_{j, k}}\left(\sup _{\mu \in \mathbf{Z}^{n}} 2^{j(\alpha+n / 2)}\left|s_{j, \mu}\right| \chi_{Q_{j, \mu}}(x)\right)^{p} d x\right\}^{1 / p} \\
& =\left\{\sum_{k \in \mathbf{Z}^{n}}\left(2^{j(\alpha+n / 2)}\left|s_{j, k}\right|\right)^{p} 2^{-j n}\right\}^{1 / p} .
\end{aligned}
$$

Fix $\varphi \in \mathcal{S}_{\infty}$. Then for every $j$ and $k$,

$$
\begin{aligned}
\left(\psi_{j, k}, \varphi\right) & =2^{-j n / 2} \int_{\mathbf{R}^{n}} \psi_{j}\left(x-k 2^{-j}\right) \varphi(x) d x \\
& =2^{-j n / 2}\left(\tilde{\psi}_{j} * \varphi\right)\left(k 2^{-j}\right)
\end{aligned}
$$

where in this proof only, we let $\tilde{\psi}(x)=\psi(-x)$. Let $N_{2}$ be a positive integer, and choose $N_{1} \in \mathbf{R}$ and $\lambda>n$ such that $N_{1}>-n-\alpha$, and $N_{1}+\lambda / \min (p, 1)-1=[n / \min (p, 1)-n-\alpha]$; this is possible since

$$
n / \min (p, 1)-n-\alpha-1<[n / \min (p, 1)-n-\alpha] .
$$

By the size estimates of Heideman type in Lemma 1.2, we have

$$
\left|\left(\psi_{j, k}, \varphi\right)\right| \leq C 2^{-j n / 2} \begin{cases}2^{-j N_{1}}(1+|k|)^{-\lambda / \min (1, p)} & \text { if } j \geq 0 \\ 2^{j N_{2}}(1+|k|)^{-\lambda / \min (1, p)} & \text { if } j<0\end{cases}
$$

(Note that in the second estimate in (2.4) we use the infinite moment condition of $\varphi$.)

To show the absolute convergence of $\sum_{j, k} s_{j, k}\left(\psi_{j, k}, \varphi\right)$, we write

$$
\begin{aligned}
\sum_{j \in \mathbf{Z}, k \in \mathbf{Z}^{n}}\left|s_{j, k}\left(\psi_{j, k}, \varphi\right)\right| & =\sum_{j \geq 0} \sum_{k \in \mathbf{Z}^{n}}\left|s_{j, k}\left(\psi_{j, k}, \varphi\right)\right|+\sum_{j \leq-1} \sum_{k \in \mathbf{Z}^{n}}\left|s_{j, k}\left(\psi_{j, k}, \varphi\right)\right| \\
& =I_{1}+I_{2} .
\end{aligned}
$$


Assume first that $p \geq 1$. Then by the above estimate (2.4) for each $\left|\left(\psi_{j, k}, \varphi\right)\right|$ and Hölder's inequality, we have

$$
\begin{aligned}
I_{1} & \leq C \sum_{j \geq 0} 2^{-j\left(N_{1}+n / 2\right)}\left(\sum_{k \in \mathbf{Z}^{n}}(1+|k|)^{-\lambda}\right)^{1 / p^{\prime}}\left(\sum_{k \in \mathbf{Z}^{n}}\left|s_{j, k}\right|^{p}(1+|k|)^{-\lambda}\right)^{1 / p} \\
& \leq C \sum_{j \geq 0} 2^{-j\left(N_{1}+n+\alpha\right)}\left(\sum_{k \in \mathbf{Z}^{n}}\left(2^{j(\alpha+n / 2)}\left|s_{j, k}\right|\right)^{p} 2^{-j n}\right)^{1 / p} \\
& \leq C\|s\|_{\dot{\mathbf{f}}_{p, \infty}^{\alpha}}<\infty
\end{aligned}
$$

by (2.3). When $p<1$, we obtain the same inequality by using the $p$-triangle inequality (instead of Hölder's inequality).

Since $\varphi$ has infinitely many vanishing moments, (2.4) holds for any $N_{2}$, and so by using a similar argument to the estimate for $I_{1}$, we can show that $I_{2} \leq C\|s\|_{\dot{\mathbf{f}}_{p, \infty}^{\alpha}}<\infty$. Since $\dot{\mathbf{f}}_{p, q}^{\alpha} \subseteq \dot{\mathbf{f}}_{p, \infty}^{\alpha}$, the proof of the lemma is complete.

Theorem 2.4. Let $-\infty<\alpha<\infty, 0<p<\infty, 0<q \leq \infty$. Assume that $\psi \in \mathcal{S}$ has $[n / \min (p, q, 1)-n-\alpha]$ vanishing moments. Then

$$
T_{\psi}: \dot{\mathbf{f}}_{p, q}^{\alpha} \rightarrow \dot{F}_{p, q}^{\alpha}
$$

(defined by (2.2)) is bounded.

Proof. Let $s \in \dot{\mathbf{f}}_{p, q}^{\alpha}$. Put

$$
f=T_{\psi}(s)=\sum_{j \in \mathbf{Z}, k \in \mathbf{Z}^{n}} s_{j, k} \psi_{j, k} .
$$

Then by Lemma 2.3, the above series converges absolutely in $\mathcal{S}^{\prime} / \mathcal{P}$.

Note that the function $\varphi$ in the definition of the Triebel-Lizorkin norms satisfies $\operatorname{supp}(\hat{\varphi}) \subseteq\{1 / 2 \leq|\xi| \leq 2\}$. Since $\varphi_{l}(x-\cdot) \in \mathcal{S}_{\infty}$ for each $l \in \mathbf{Z}$ and $x \in \mathbf{R}^{n}$, the convergence in $\mathcal{S}^{\prime} / \mathcal{P}$ implies the pointwise representation

$$
\left(\varphi_{l} * f\right)(x)=\sum_{j \in \mathbf{Z}, k \in \mathbf{Z}^{n}} s_{j, k}\left(\psi_{j, k} * \varphi_{l}\right)(x)
$$

(where the convergence of the series on the right-hand side is also absolute). It follows that

$$
\begin{aligned}
\left|\left(\varphi_{l} * f\right)(x)\right| \leq & \sum_{j \geq l} \sum_{k \in \mathbf{Z}^{n}}\left|s_{j, k}\right|\left|\left(\psi_{j, k} * \varphi_{l}\right)(x)\right| \\
& +\sum_{j \leq l-1} \sum_{k \in \mathbf{Z}^{n}}\left|s_{j, k}\right|\left|\left(\psi_{j, k} * \varphi_{l}\right)(x)\right| \\
= & I_{1}(x, l)+I_{2}(x, l) .
\end{aligned}
$$


Set $r=\min (p, q)$. Pick a non-negative integer $N_{2}$ such that $N_{2}-$ $\alpha>0$. Choose $\lambda>n$ and $N_{1} \in \mathbf{R}$ such that $N_{1}>-n-\alpha$ and $N_{1}+\lambda / \min (r, 1)-1=[n / \min (1, r)-n-\alpha]$. Since for all $j$ and $k$,

$$
\left(\psi_{j, k} * \varphi_{l}\right)(x)=2^{-j n / 2}\left(\psi_{j} * \varphi_{l}\right)\left(x-k 2^{-j}\right),
$$

$\psi$ has $[n / \min (1, r)-n-\alpha]$ vanishing moments, and $\varphi$ has infinitely many vanishing moments, Lemma 1.2 implies that

$$
\begin{aligned}
& \left|\left(\psi_{j, k} * \varphi_{l}\right)(x)\right| \leq \\
& \quad C 2^{-j n / 2} \begin{cases}2^{l n} 2^{N_{1}(l-j)}\left(1+2^{j}\left|x-k 2^{-j}\right|\right)^{-\lambda / \min (1, r)}, & j \geq l \\
2^{j n} 2^{N_{2}(j-l)}\left(1+2^{l}\left|x-k 2^{-j}\right|\right)^{-\lambda / \min (1, r)}, & j<l .\end{cases}
\end{aligned}
$$

By using the above estimate and an argument similar to the proof of Lemma 2.3, we obtain

$$
I_{1}(x, l) \leq C \sum_{j \geq l} 2^{-j\left(N_{1}+n / 2\right)} 2^{l\left(N_{1}+n\right)}\left(\sum_{k \in \mathbf{Z}^{n}}\left|s_{j, k}\right|^{r}\left(1+2^{j}\left|x-k 2^{-j}\right|\right)^{-\lambda}\right)^{1 / r},
$$

where we also used the obvious estimate:

$$
\sum_{k \in \mathbf{Z}^{n}}\left(1+2^{j}\left|x-k 2^{-j}\right|\right)^{-\lambda}=\sum_{k \in \mathbf{Z}^{n}}\left(1+\left|2^{j} x-k\right|\right)^{-\lambda} \approx \sum_{k \in \mathbf{Z}^{n}}(1+|k|)^{-\lambda}
$$

for all $j \in \mathbf{Z}$ and $x \in \mathbf{R}^{n}$.

Next, note that

$$
\begin{aligned}
& \left(\sum_{k \in \mathbf{Z}^{n}}\left|s_{j, k}\right|^{r}\left(1+2^{j}\left|x-k 2^{-j}\right|\right)^{-\lambda}\right)^{1 / r}= \\
& \sum_{m \in \mathbf{Z}^{n}}\left(\sum_{k \in \mathbf{Z}^{n}}\left|s_{j, k}\right|^{r}\left(1+2^{j}\left|x-k 2^{-j}\right|\right)^{-\lambda}\right)^{1 / r} \chi_{Q_{j, m}}(x) \\
& \leq C \sum_{m \in \mathbf{Z}^{n}}\left(\sum_{k \in \mathbf{Z}^{n}}\left|s_{j, k}\right|^{r}\left(1+2^{j}\left|m 2^{-j}-k 2^{-j}\right|\right)^{-\lambda}\right)^{1 / r} \chi_{Q_{j, m}}(x) \\
& \quad=C \sum_{m \in \mathbf{Z}^{n}} s_{j, m}^{*} \chi_{Q_{j, m}}(x),
\end{aligned}
$$

as $\left(1+2^{j}\left|x-k 2^{-j}\right|\right) \approx\left(1+2^{j}\left|m 2^{-j}-k 2^{-j}\right|\right)$, for all $x \in Q_{j, m}$. It follows that

$$
I_{1}(x, l) \leq C \sum_{j \geq l} 2^{(l-j)\left(n+N_{1}\right)} \sum_{m \in \mathbf{Z}^{n}} s_{j, m}^{*} \chi_{j, m}(x)
$$


Therefore, if $1 \leq q$, then using Hölder's inequality for the sum with respect to $j$, we obtain

$$
\begin{aligned}
\sum_{l \in \mathbf{Z}}\left(2^{l \alpha} I_{1}(x, l)\right)^{q} & \leq C \sum_{l \in \mathbf{Z}}\left\{\sum_{j \geq l} 2^{(l-j)\left(n+N_{1}+\alpha\right)}\left(\sum_{m \in \mathbf{Z}^{n}} 2^{j \alpha} s_{j, m}^{*} \chi_{j, m}(x)\right)^{q}\right\} \\
& =C \sum_{l \in \mathbf{Z}} \sum_{j \geq l} 2^{(l-j)\left(n+N_{1}+\alpha\right)} \sum_{m \in \mathbf{Z}^{n}}\left(2^{j \alpha} s_{j, m}^{*} \chi_{j, m}(x)\right)^{q} \\
& =C \sum_{j \in \mathbf{Z}}\left(\sum_{l \leq j} 2^{(l-j)\left(n+N_{1}+\alpha\right)}\right) \sum_{m \in \mathbf{Z}^{n}}\left(2^{j \alpha} s_{j, m}^{*} \chi_{j, m}(x)\right)^{q} \\
& \leq C \sum_{j \in \mathbf{Z}} \sum_{m \in \mathbf{Z}^{n}}\left(2^{j \alpha} s_{j, m}^{*} \chi_{j, m}(x)\right)^{q}
\end{aligned}
$$

The last inequality in (2.7) and Lemma 1.1 imply that

$$
\begin{aligned}
\left\|\left(\sum_{l \in \mathbf{Z}}\left(2^{l \alpha} I_{1}(x, l)\right)^{q}\right)^{1 / q}\right\|_{p} & \leq C\left\|s^{*}\right\|_{\dot{\mathbf{f}}_{p, q}^{\alpha}} \\
& \leq C\|s\|_{\dot{\mathbf{f}}_{p, q}^{\alpha}} .
\end{aligned}
$$

Since we also have (2.7) when $q<1$ (by using the $q$-triangle inequality instead of Hölder's inequality), we obtain (2.8) for all $q$.

On the other hand, using the second estimate in (2.6) and an argument similar to the estimate for $I_{1}(x, l)$, we have

$$
\left\|\left(\sum_{l \in \mathbf{Z}}\left(2^{l \alpha} I_{2}(x, l)\right)^{q}\right)^{1 / q}\right\|_{p} \leq C\|s\|_{\dot{\mathbf{f}}_{p, q}^{\alpha}} .
$$

Thus, the conclusion of the theorem follows from (2.5), (2.8) and (2.9).

Remarks. (i) Since the constant $C$ in (2.6) has the form

$$
C=C(n) p_{N}(\psi)
$$

for a sufficiently large non-negative integer $N$ (depending on $N_{1}, \lambda, n, p, q$ ), a quantitative version of Theorem 2.4 is that the inequality

$$
\left\|T_{\psi}(s)\right\|_{\dot{F}_{p, q}^{\alpha}} \leq C(\alpha, n, p, q) p_{N}(\psi)\|s\|_{\dot{\mathbf{f}}_{p, q}^{\alpha}}
$$

holds for every $s \in \dot{\mathbf{f}}_{p, q}^{\alpha}$. This remark is important in the next section.

(ii) A careful examination of the proofs shows that these can be modified to prove that Lemma 2.3 and Theorem 2.4 hold when each $\psi_{j, k}$ is replaced by $\psi_{j, k}^{(a)}$, where $\psi_{j, k}^{(a)}(x)=2^{j n / 2} \psi\left(2^{j} x-a(k) k\right)$, and $0<$ $c_{1} \leq a(k) \leq c_{2}<\infty$ for some positive constants $c_{1}, c_{2}$ and all $k \in \mathbf{Z}$. This remark is used in the proof of the last assertion of Theorem 3.2. 
(iii) Theorem 2.4 can also be proved by using the molecular decompositions of the Triebel-Lizorkin spaces [8, Theorem 3.5]. However, the proof of this decomposition in [8] used the equivalence (3.4) which we aim to prove by using our Theorems 2.4 and 2.2. We hope that our direct and simple approach could be useful in some other circumstances.

\section{INVERTIBILITY AND NORM-EQUIVALENCE}

First we recall a main result of [8], namely Theorem 2.2 in that paper:

If $\varphi$ and $\psi$ are functions in $\mathcal{S}$ which satisfy

$$
\begin{array}{r}
\operatorname{supp}(\hat{\varphi}), \operatorname{supp}(\hat{\psi}) \subseteq\{1 / 2 \leq|\xi| \leq 2\}, \\
|\hat{\varphi}(\xi)|,|\hat{\psi}(\xi)| \geq c>0, \quad \text { if } 3 / 5 \leq|\xi| \leq 5 / 3,
\end{array}
$$

and

$$
\sum_{j \in \mathbf{Z}} \overline{\hat{\varphi}\left(2^{j} \xi\right)} \hat{\psi}\left(2^{j} \xi\right)=1, \quad|\xi| \neq 0
$$

then

$$
\|f\|_{\dot{F}_{p, q}^{\alpha}} \approx\left\|S_{\varphi}(f)\right\|_{\dot{\mathbf{f}}_{p, q}^{\alpha}}
$$

for all $\alpha, p, q$, and all $f \in \mathcal{S}^{\prime} / \mathcal{P}$.

Note that if $\psi \in \mathcal{S}$ satisfies (3.1) and (3.2), then there exists $\varphi \in \mathcal{S}$ such that (3.1)-(3.3) are satisfied.

The proof of the (3.4) in [8] uses certain equivalence which seems true only for entire functions of exponential type. We give below a proof based on the results in Section 2 and an identity in [7].

Assume that $\varphi$ and $\psi$ have appropriate vanishing moments. Then, by Theorems 2.4 and 2.2 ,

$$
\begin{aligned}
\left\|\left(T_{\psi} \circ S_{\varphi}\right)(f)\right\|_{\dot{F}_{p, q}^{\alpha}} & \leq C\left\|S_{\varphi}(f)\right\|_{\dot{\mathbf{f}}_{p, q}^{\alpha}} \\
& \leq C\|f\|_{\dot{F}_{p, q}^{\alpha}} .
\end{aligned}
$$

Therefore, if (3.1)-(3.3) are satisfied, then by (3.1) both $\varphi$ and $\psi$ have infinitely many vanishing moments, so that (3.5) implies half of the equivalence (3.4). The other half of this equivalence follows from the first inequality in (3.5) and the identity

$$
\left(T_{\psi} \circ S_{\varphi}\right)(f)=\sum_{j \in \mathbf{Z}} \sum_{k \in \mathbf{Z}^{n}}\left\langle f, \varphi_{j, k}\right\rangle \psi_{j, k}=f \quad \text { in } \mathcal{S}^{\prime} / \mathcal{P} .
$$

(See [7, Lemma 2.1].) The proof of (3.6) depends crucially on a sampling theorem in which the assumption (3.1) is critical (see [7, Lemma 2.1] or [9, Lemma 6.10] for this sampling theorem). 
By a similar argument, we can prove the following theorem in which we require a slightly less stringent Tauberian condition than condition $(3.2)$.

Theorem 3.1. Let $-\infty<\alpha<\infty, 0<p<\infty$ and $0<q \leq \infty$. Let $\psi \in \mathcal{S}$ have $[n / \min (1, p, q)-n-\alpha]$ vanishing moments. Assume that $\psi$ satisfies the following conditions:

(a) $\operatorname{supp} \hat{\psi} \subset \mathbf{T}_{n}:=\left\{\xi: \max _{j=1, \ldots, n}\left|\xi_{j}\right|<\pi\right\}$;

(b) For each $|\xi|=1$, there exist positive numbers $0<a<b<\infty$ such that $2 a \leq b$ and for which

$$
\hat{\psi}(t \xi) \neq 0 \quad \text { for all } a \leq t \leq b .
$$

The there exists $\varphi \in \mathcal{S}$ such that $\operatorname{supp} \hat{\varphi}$ is contained in $\mathbf{T}_{n}$, and that (3.4) and (3.6) hold for every $f \in \mathcal{S}^{\prime} / \mathcal{P}$. Moreover, if $q<\infty$, then the series in (3.6) converges also in the $\dot{F}_{p, q}^{\alpha}$-norm.

Proof. As observed in [4, page 541], the Tauberian condition (b) implies that there exists $\varphi \in \mathcal{S}$ such that $\hat{\varphi}$ is supported in an annulus about the origin and that (3.3) holds. By multiplying $\hat{\varphi}$ by a cut-off function and using (a) we may assume that $\hat{\varphi}$ is supported in $\mathbf{T}_{n}$. Since $\varphi$ has infinitely many vanishing moments and $\psi$ has the right number of vanishing moments (see Theorem 2.4), we can use Theorems 2.2 and 2.4 to obtain (3.5). Since both $\hat{\psi}$ and $\hat{\varphi}$ are supported in $\mathbf{T}_{n}$ and (3.3) holds, we apply the sampling theorem [9, Lemma 6.10] to obtain (3.6). We then deduce (3.4) as before. The norm-convergence when $q<\infty$ follows from Theorem 2.4 .

We next discuss the general case where $\varphi$ and $\psi$ have appropriate vanishing moments, and (3.3) is satisfied. We are interested in the equivalence (3.4). By the arguments preceding Theorem 3.1, we would have this equivalence if we can prove (3.6). Applying Theorem 2.2 to the spaces $\dot{F}_{2,2}^{0}=L^{2}$ and $\dot{\mathbf{f}}_{2,2}^{0}=\ell^{2}$, we obtain

$$
\begin{gathered}
\sum_{j \in \mathbf{Z}} \sum_{k \in \mathbf{Z}^{n}}\left|\left\langle\varphi_{j, k}, f\right\rangle\right|^{2} \leq C\|f\|_{2}^{2}, \\
\sum_{j \in \mathbf{Z}} \sum_{k \in \mathbf{Z}^{n}}\left|\left\langle\psi_{j, k}, f\right\rangle\right|^{2} \leq C\|f\|_{2}^{2} .
\end{gathered}
$$

Assume furthermore that $\varphi$ and $\psi$ satisfy the $t_{q}$-condition:

$t_{q}(\xi)=\sum_{m=0}^{\infty} \hat{\varphi}\left(2^{m} \xi\right) \overline{\hat{\psi}\left(2^{m}(\xi+2 \pi q)\right)}=0 \quad$ for all $\xi \in \mathbf{R}^{n}$ and all $q \in \mathcal{O}^{n}$, 
where $\mathcal{O}^{n}$ denotes the set of $q \in \mathbf{Z}^{n}$ such that at least one component of $q$ is odd. Then (3.3), (3.7), (3.8) and (3.9), together with [6, Theorem 4.9 ], imply that

$$
f=\sum_{j \in \mathbf{Z}} \sum_{k \in \mathbf{Z}^{n}}\left\langle f, \varphi_{j, k}\right\rangle \psi_{j, k}=\sum_{j \in \mathbf{Z}} \sum_{k \in \mathbf{Z}^{n}}\left\langle f, \psi_{j, k}\right\rangle \varphi_{j, k}
$$

in $L^{2}$. Hence it follows easily that (3.6) holds for every $f \in \dot{F}_{p, q}^{\alpha}$.

Thus, we have proved that the norm-equivalence (3.4) holds when $\varphi$ and $\psi$ have appropriate vanishing moments, and (3.3) and (3.9) are satisfied. Therefore, for a given function $\psi \in \mathcal{S}$ with an appropriate moment condition, it is of interest to find simply stated condtions on $\psi$ so that there is $\varphi \in \mathcal{S}$ for which (3.3) and (3.9) hold. Although there is a simple condition on $\psi$ to ensure the existence of $\varphi$ such that (3.3) holds (see (i) of the Remarks to the next theorem), a satisfactory condition to imply (3.9) remains elusive. We note in the band-limited case that (3.1) trivially implies (3.9).

As for the equivalence (3.4) in the general case we have the following theorem, which is the main result of this paper.

Theorem 3.2. Let $-\infty<\alpha<\infty, 0<p<\infty, 0<q \leq \infty$. Suppose that $\psi \in \mathcal{S}$ has $[n / \min (p, q, 1)-n-\alpha]$ vanishing moments, and that it satisfies the dyadic Tauberian condition; i.e., there is $\varphi \in \mathcal{S}$ such that $\operatorname{supp}(\hat{\varphi})$ is contained in an annulus about the origin and that (3.3) holds. Then there exist $L \in \mathbf{Z}$ and $\Phi \in \mathcal{S}$ such that for each $l \leq L$,

$$
\|f\|_{\dot{F}_{p, q}^{\alpha}} \approx\left\|S_{\varphi_{l} * \Phi}(f)\right\|_{\dot{\mathbf{f}}_{p, q}^{\alpha}}
$$

for all $f \in \mathcal{S}^{\prime} / \mathcal{P}$, and $T_{\psi_{l}} \circ S_{\varphi_{l} * \Phi}$ is invertible on $\dot{F}_{p, q}^{\alpha}$. Moreover, if $q<\infty$, then the linear span of

$$
\left\{\psi\left(2^{j} x-k 2^{l}\right): k \in \mathbf{Z}^{n}, j \in \mathbf{Z}\right\}
$$

is dense in $\dot{F}_{p, q}^{\alpha}$.

Proof. By dilating $\varphi$ and $\psi$ if necessary, we may assume that $\operatorname{supp}(\hat{\varphi}) \subseteq$ $\{|\xi| \leq 2\}$. Choose $\Phi \in \mathcal{S}$ such that $\hat{\Phi}$ is non-negative and radial, $\operatorname{supp}(\hat{\Phi}) \subseteq\{|\xi| \leq 3\}$, and $\hat{\Phi}=1$ on $\{|\xi| \leq 2\}$. Let $l \leq 0$. Then, since $\tilde{\varphi}_{l} * \psi_{l}=\left(\varphi_{l} * \Phi\right)^{\sim} * \psi_{l} * \Phi$, the condition (3.3) implies that

$$
f=\sum_{j \in \mathbf{Z}}\left(\varphi_{l} * \Phi\right)_{j}^{r} *\left(\psi_{l} * \Phi\right)_{j} * f
$$


in $\mathcal{S}^{\prime} / \mathcal{P}$. By this representation and the assumptions on the support of $\hat{\Phi}$, together with the sampling theorem [9, Lemma 6.10], we have

$$
\begin{aligned}
f & =\sum_{j \in \mathbf{Z}} \sum_{k \in \mathbf{Z}^{n}}\left\langle f,\left(\varphi_{l} * \Phi\right)_{j, k}\right\rangle\left(\psi_{l} * \Phi\right)_{j, k} \\
& =\left(T_{\psi_{l} * \Phi} \circ S_{\varphi_{l} * \Phi}\right)(f) .
\end{aligned}
$$

Since $\psi_{l} * \Phi$ has the same vanishing moments as $\psi$, and $\varphi_{l} * \Phi$ has infinitely many vanishing moments, Theorems 2.2 and 2.4 then imply (3.10) for all $f \in \mathcal{S}^{\prime} / \mathcal{P}$. It also follows from the above and the quantitative versions of Theorems 2.2 and 2.4 (see (2.1) and (2.10) that

$$
\begin{gathered}
\left\|f-\left(T_{\psi_{l}} \circ S_{\varphi_{l} * \Phi}\right)(f)\right\|_{\dot{F}_{p, q}^{\alpha}}=\left\|\left(T_{\left(\psi_{l} * \Phi-\psi_{l}\right)} \circ S_{\varphi_{l} * \Phi}\right)(f)\right\|_{\dot{F}_{p, q}^{\alpha}} \\
\leq C p_{N}\left(\psi_{l} * \Phi-\psi_{l}\right) p_{N}\left(\varphi_{l} * \Phi\right)\|f\|_{\dot{F}_{p, q}^{\alpha}} .
\end{gathered}
$$

By the assumptions on $\varphi$ and $\Phi$, there is $N_{1}$ (depending on $N$ ), and for each $N_{2}>0$, there is a constant $C$ which may depend on $N_{1}, N_{2}$ but not on $l$ such that

$$
\begin{aligned}
p_{N}\left(\varphi_{l} * \Phi\right) & \leq C 2^{-l N_{1}} \\
p_{N}\left(\psi_{l} * \Phi-\psi_{l}\right) & \leq C 2^{l N_{2}} .
\end{aligned}
$$

These estimates and (3.11) imply that, for every $\epsilon>0$, there is a non-positive integer $L_{\epsilon}$ such that

$$
\left\|f-\left(T_{\psi_{l}} \circ S_{\varphi_{l} * \Phi}\right)(f)\right\|_{\dot{F}_{p, q}^{\alpha}} \leq \epsilon\|f\|_{\dot{F}_{p, q}^{\alpha}}
$$

for all $l \leq L_{\epsilon}$. Let $\rho=\min (1, p, q)$. Then (3.12) implies that there exists $L \in \mathbf{Z}$ such that for each $l \leq L$, and

$$
U_{l}(f)=I(f)-\left(T_{\psi_{l}} \circ S_{\varphi_{l} * \Phi}\right)(f)=f-\left(T_{\psi_{l}} \circ S_{\varphi_{l} * \Phi}\right)(f),
$$

we have $\left\|U_{l}(f)\right\|_{\dot{F}_{p, q}^{\alpha}}^{\rho} \leq(1 / 2)\|f\|_{\dot{F}_{p, q}^{\alpha}}^{\rho}$, and by iterating

$$
\left\|U_{l}^{m}(f)\right\|_{\dot{F}_{p, q}^{\alpha}}^{\rho} \leq(1 / 2)^{m}\|f\|_{\dot{F}_{p, q}^{\alpha}}^{\rho},
$$

for all $f \in \dot{F}_{p, q}^{\alpha}$, and $m=1,2, \ldots$ Since $\|\cdot\|_{\dot{F}_{p, q}^{\alpha}}$ is a $\rho$-norm, $\|f+g\|_{\dot{F}_{p, q}^{\alpha}}^{\rho} \leq$ $\|f\|_{\dot{F}_{p, q}^{\alpha}}^{\rho}+\|g\|_{\dot{F}_{p, q}^{\alpha}}^{\rho}$, and $\dot{F}_{p, q}^{\alpha}$ is complete, (3.13) and a well-known theorem in functional analysis [13, Theorem 2.8] imply that $I+U_{l}+U_{l}^{2}+U_{l}^{3}+\cdots$ is a bounded operator on $\dot{F}_{p, q}^{\alpha}$. This last operator is the inverse of $I-U_{l}=T_{\psi_{l}} \circ S_{\varphi_{l} * \Phi}$.

To prove the last assertion of the theorem, fix $l \leq L$ such that $T_{\psi_{l}} \circ S_{\varphi_{l} * \Phi}$ is invertible. Let $f \in \dot{F}_{p, q}^{\alpha}$. Then there exists $g \in \dot{F}_{p, q}^{\alpha}$ such 
that

$$
\begin{aligned}
f=\left(T_{\psi_{l}} \circ S_{\varphi_{l} * \Phi}\right)(g) & =\sum_{j, k}\left(S_{\varphi_{l} * \Phi}(g)\right)_{j, k}\left(\psi_{l}\right)_{j, k} \\
& =\sum_{j, k}\left(S_{\varphi_{l} * \Phi}(g)\right)_{j, k} 2^{j / 2} 2^{l n} \psi\left(2^{j+l} x-k 2^{l}\right) \\
& =\sum_{j, k}\left(S_{\varphi_{l} * \Phi}(g)\right)_{j-l, k} 2^{j / 2} 2^{l n} \psi\left(2^{j} x-k 2^{l}\right) .
\end{aligned}
$$

By Lemma 2.3 the series in the right-hand side of the above converges in $\mathcal{S}^{\prime} / \mathcal{P}$, but if $q<\infty$, then Theorem 2.4 implies that we have also convergence in $\dot{F}_{p, q}^{\alpha}$. Thus we obtain the assertion on the density of the spanning set and complete the proof of the theorem.

REMARKs. (i) The condition (3.3) and its use in the theory of function spaces are well-known. However, the terminology "dyadic Tauberian condition" was coined in [4] in which the authors also observed that this condition holds if condition (b) of Theorem 3.1 is satisfied.

(ii) Our results should be compared to [8, Theorem 4.2] where, instead of the dyadic Tauberian condition the authors assumed that $|\hat{\psi}(\xi)| \geq c>0$ if $1 / 2 \leq|\xi| \leq 2$, and, in addition to the moment condition the authors assumed certain size conditions and Hölder's estimates on $\psi$ and its derivatives. The proof in [8] uses the molecular decompostions of the Triebel-Lizorkin spaces and is considerably more complicated than our proof of Theorem 3.2. Their method could be applied to the case where $\psi$ may not be in $\mathcal{S}$. Actually, all our results are also valid in the case when $\varphi$ or $\psi$ is not in $\mathcal{S}$, but they are sufficiently smooth and satisfy appropriate moment conditions and size conditions (of the form $p_{N}(\varphi)<\infty$ and/or $p_{N}(\psi)<\infty$ ). The proofs are the same. We have restricted our theorems to the case $\varphi$ and $\psi$ in $\mathcal{S}$ for simplicity of presentation. We refer to [2, Section 6, Remark (b)] for a similar remark on Lemma 1.3, that this lemma holds for some $\varphi$ not in $\mathcal{S}$.

(iii) Set $V_{l}=T_{\psi_{l}} \circ S_{\varphi_{l} * \Phi}$. Then $g=V_{l}^{-1}(f)$ in the above proof and, as in [8], (3.14) can be written as

$$
f=\sum_{j, k}\left\langle V_{l}^{-1} f,\left(\varphi_{l} * \Phi\right)_{j, k}\right\rangle\left(\psi_{l}\right)_{j, k}=\sum_{j, k}\left\langle f,\left(V_{l}^{-1}\right)^{*}\left(\left(\varphi_{l} * \Phi\right)_{j, k}\right)\right\rangle\left(\psi_{l}\right)_{j, k} .
$$

This should be compared with the results in [10] in which the authors prove that, under "minimal" conditions on the function $\psi$, and for $1 \leq p, q<\infty$, there exist $r>1, s>0$ and a family of functions $\left\{\rho^{j, k}\right\}$ 
(the so-called molecules), such that

$$
f=\sum_{j, k}\left\langle f, \rho^{j, k}\right\rangle \psi_{j, k}^{(r, s)}
$$

for every $f \in \dot{F}_{p, q}^{\alpha}$, where $\psi_{j, k}^{(r, s)}(x)=r^{j n / 2} \psi\left(r^{j} x+s k\right)$. The explicit values of $r$ and $s$ are unknown. The proofs in [10] use the molecular decompositions of the Triebel-Lizorkin spaces in [8] and the subtle theory of Calderón-Zygmund operators. The results in [10] are probably the most comprehensive ones in the theory of molecular and frame decompositions for these spaces.

(iv) A final point of interest of our approach: The simplicity of the proofs of our results makes it possible to explicitly display the dependence on $\alpha, p, q$ and $\psi$ of the various constants appearing in each proof. This may be useful in applications, for instance, when one wishes to estimate the number $L_{\epsilon}$ in the proof of Theorem 3.2 so that (3.12) holds.

We next discuss a special case of Theorem 3.2, which has partially motivated our work. In $\mathbf{R}$ consider the "Mexican hat" function

$$
\psi(x)=-\frac{d^{2}}{x^{2}}\left[e^{-x^{2} / 2}\right]=\left(1-x^{2}\right) e^{-x^{2} / 2} .
$$

This function is in $\mathcal{S}(\mathbf{R})$ and has one vanishing moment. Since $\hat{\psi}(\xi) \neq 0$ for all $\xi \neq 0, \psi$ also satisfies the dyadic Tauberian condition. If we apply Theorem 3.2 to the Hardy space $H^{p}(\mathbf{R})=\dot{F}_{p, 2}^{0}(\mathbf{R})$, then in this case, $[1 / \min (p, 2,1)-1] \leq 1$ when $p>1 / 3$. Therefore, we obtain the following theorem:

Theorem 3.3. Let $\psi$ be the Mexican hat function given by (3.15), and $p>1 / 3$. Then there exists $L \in \mathbf{Z}$ such that for each $l \leq L$, the linear span of

$$
\left\{\psi\left(2^{j} x-k 2^{l}\right): j, k \in \mathbf{Z}\right\}
$$

is dense in $H^{p}(\mathbf{R})$.

Note that, since $H^{p}(\mathbf{R})=L^{p}(\mathbf{R})$ if $1<p<\infty$, Theorem 3.3 is closely related to a question posed by Y. Meyer in [11] where he asked whether or not the linear span of

$$
\left\{\psi\left(2^{j} x-k\right): j, k \in \mathbf{Z}\right\}
$$

is dense in $L^{p}(\mathbf{R}), p \neq 2$. It was known from a result by I. Daubechies that $\left\{\psi_{j, k}: j, k \in \mathbf{Z}\right\}$ is a frame in $L^{2}(\mathbf{R})$ (see [5], [11]).

From the proof of Theorem 3.2, it is very likely that the number $L$ in Theorem 3.3 is negative, and therefore Theorem 3.3 does not resolve 
the Meyer's problem; we need "oversampling" to obtain a spanning set. This "oversampling" phenomenon also occurs in [10] in which the authors show that, for each $1 \leq p<\infty$, there exist (sufficiently small) $r>1$ and $s>0$ for which the linear span of

$$
\left\{\psi\left(r^{j} x-k s\right): j, k \in \mathbf{Z}\right\}
$$

is dense in $H^{p}(\mathbf{R})$. However, the explicit values of $r$ and $s$ are not known.

A significant open problem is to investigate the explicit relationship between the various conditions on a function $\psi$ and the dilation factor and sampling points in the spanning set. When $\int_{\mathbf{R}^{n}} \psi(x) d x \neq 0$, a rather complete solution for the spanning problem in Lebesgue and Sobolev spaces has been given given in [1].

We end this paper by a remark. Since the main tools in our approach, Lemmas 1.1 and 1.3, hold for weighted spaces, all our main results are valid in the weighted setting in which the weight function is in the Muckenhoupt class $A_{\infty}$, and moreover, they are also valid for the weighted Besov-Lipschitz spaces. By the same reasons, these results (except Theorem 3.3 and the density result in Theorem 3.2) hold in the case $p=\infty$. We leave the details to the interested reader.

\section{REFERENCES}

[1] H.-Q. Bui and R.S. Laugesen, Spanning and sampling in Lebesgue and Sobolev spaces, Research Report UCDMS2004/8, 64 pages, May 2004.

[2] H.-Q. Bui, M. Paluszyński and M.H. Taibleson, A maximal function characterization of weighted Besov-Lipschitz and Triebel-Lizorkin spaces, Studia Math. 119(1996), 219-246.

[3] H.-Q. Bui, M. Paluszyński and M.H. Taibleson, Characterization of the BesovLipschitz and Triebel-Lizorkin spaces. The case $q<1$, J. Fourier Anal. Appl. 3(1997), 837-846.

[4] H.-Q. Bui and M.H. Taibleson, The characterization of the Triebel-Lizorkin spaces for $p=\infty$, J. Fourier Anal. Appl. 6(2000), 537-550.

[5] I. Daubechies, Ten lectures on wavelets, CBS-NSF Regional Conferences in Applied Mathematics, No.61, SIAM, 1992.

[6] M. Frazier, G. Garrigós, K. Wang and G. Weiss, A characterization of functions that generate wavelet and related expansions, J. Fourier Anal. Appl. 3(1997), 883-906.

[7] M. Frazier and B. Jawerth, Decomposition of Besov spaces, Indiana Univ. Math. J. 34(1985), 777-799.

[8] M. Frazier and B. Jawerth, A discrete transform and decomposition of distribution spaces, J. Funct. Anal. 93(1990), 34-170.

[9] M. Frazier, B. Jawerth and G. Weiss, Littlewood-Paley theory and the study of function spaces, CBMS - AMS, 79(1991). 
[10] J.E. Gilbert, Y.S. Han, J.A. Hogan, J.D. Lakey, D. Weiland and G. Weiss, Smooth molecular decompositions of function spaces and singular integral operators, Memoirs Amer. Math. Soc. 156(2002), No.742.

[11] Y. Meyer, Ondelettes et opérateurs I, Herman, Paris, 1990.

[12] J. Peetre, On spaces of Triebel-Lizorkin type, Ark. Mat. 13(1975), 123-130.

[13] W. Rudin, Functional analysis, McGraw-Hill, New York, 1973.

[14] H. Triebel, Theory of function spaces, Birkhäuser, Basel, 1983.

Department of Mathematics, University of Canterbury, Christchurch 8020, New Zealand.

E-mail address: q.bui@math.canterbury.ac.nz

Institute of Mathematics, University of Wroclaw, 50-384 Wroclaw, POLAND.

E-mail address: mpal@math.uni.wroc.pl 